\title{
Overview of Borophene as a Potential Candidate in 2D Materials Science for the Energy Applications
}

\author{
Niket Powar, Rajkumar Pandav* \\ Department of Chemistry, Yashawantrao Chavan Warana Mahavidyalaya, Warananager, Shivaji University, Kolhapur, \\ Maharashtra, India-416113
}

\begin{abstract}
Receive Date: 25 August 2019, Revise Date: 07 October 2019, Accept Date: 08 October 2019
Abstract:

Energy problem is one of the serious concerns in modern society; therefore, we have to take hastily an effective action. Hence, researchers are looking for some attractive materials with low-cost, lightweight, and environmentally effective. Recently, 2D materials have taken notable recognition in the field of materials science for multiple energy application, because of its unique electronic and optical properties; and borophene is one of the 2D material which is commendatories than graphene. However, it has not much experimentally explored yet. This review discusses the synthesis process of borophene and discussed energy-related application such as energy storage, optoelectronic, photocatalytic activity, and hydrogen storage. Moreover, this work provides a summary of each application that could help to understand the importance of borophene materials for energy applications.

DOI: $10.33945 /$ SAMI/JCR.2019.4.3

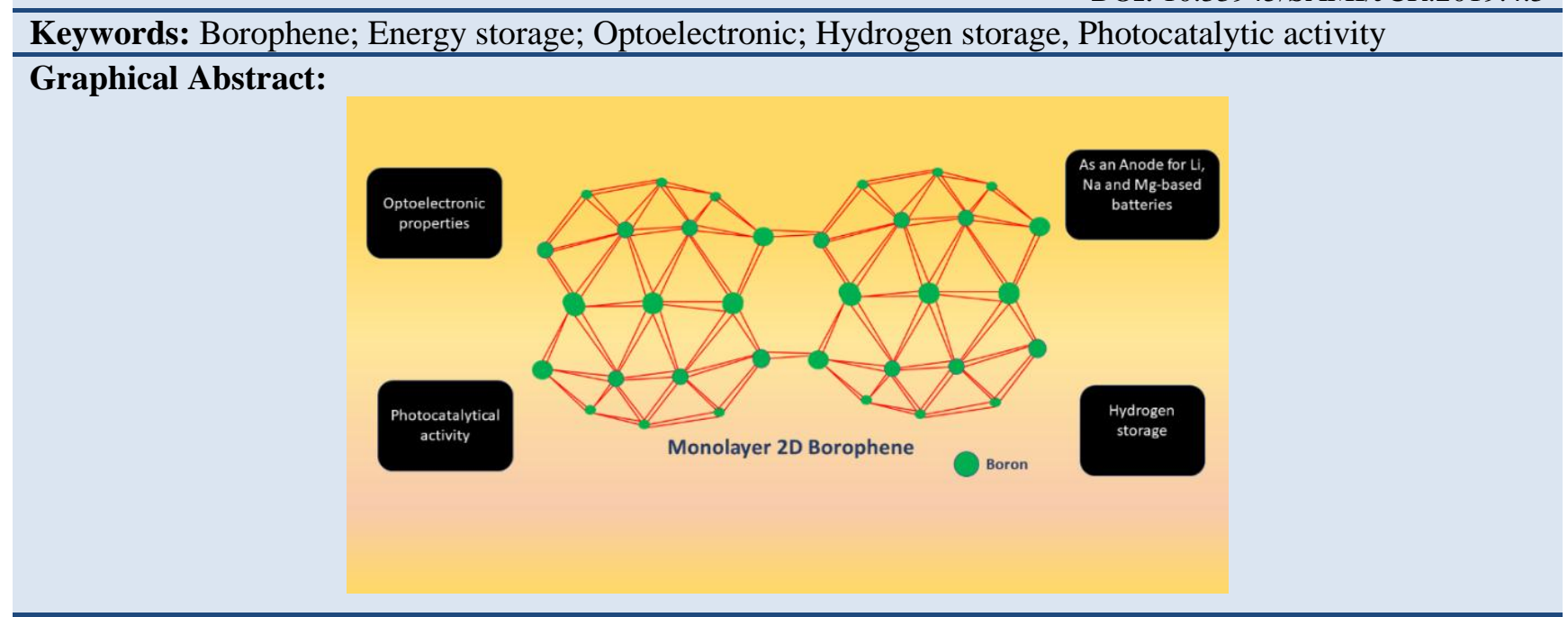

Biography:
Niket Powar has completed M.Sc in Organic chemistry from Yashwantarao
Chavan Institute of Science, Satara, Shivaji University, Kolhapur, Maharashtra,
India. Afterwards, he has worked as Junior Research Fellow (JRF) in Ahmedabad
university at Gujarat, India. Whereas, he has worked on the synthesis of bimetallic
nanocomposite and demonstrated the catalytical activity for an organic
transformation reaction. Recently, he has pursued M.Tech in nanotechnology and
renewable energy and as part of M.Tech project, worked on the energy-related
topic including the 2D materials. Moreover, his area of research interest is in the
photocatalytic activity, water splitting, quantum dots based solar cell and 2D based
materials for energy application. Now he is working as a research co-worker with
Dr Rajkumar Pandav.
\end{abstract}




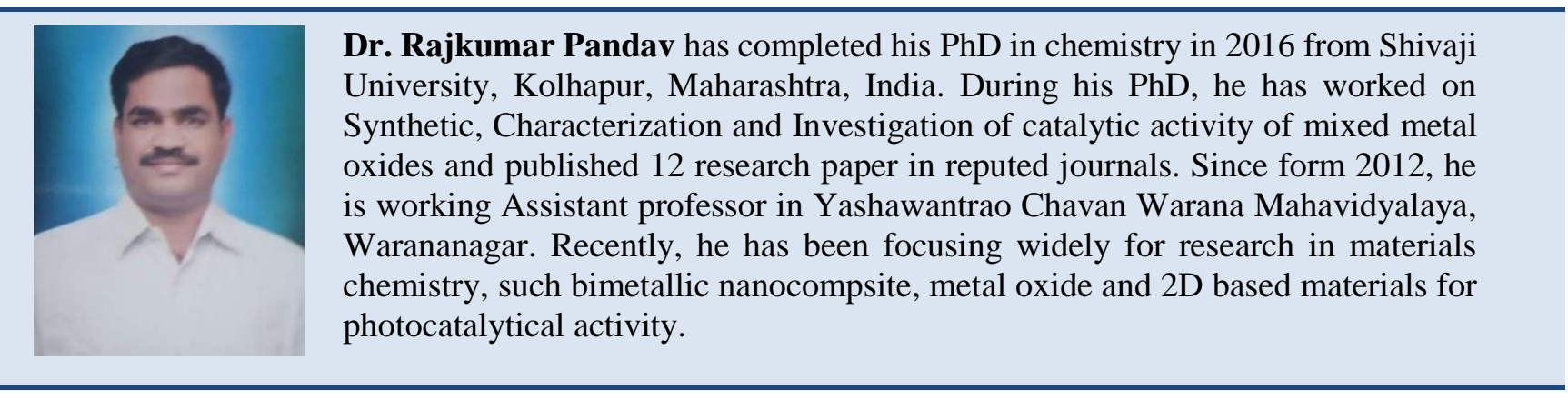

\section{Introduction}

Nowadays, an emerging trend of 2D based inorganic materials [1-5] have been expanded hastily for application of energy conversion [6,7] and storage, $[8,9]$ After graphene was discovered, researchers have found astonishing electronic [10,11], mechanical $[12,13]$ and thermal [14] properties. Afterwards, discovered several 2D based materials such as Silicene [15,16] Germanene [17-19], Phosphorene [20,21], Arsenene [22,23], Antimonene [24] and transition metal dichalcogenides [25-27]. In Periodic table Boron comes nearest to Carbon element and Boron has an ability to form of polyhedral cluster structure, impact of that in 1996, Boustani [28] has done some computerized based calculation and proved that quasi planner cluster of Boron has remarkable properties resemble to the Graphite, we will discuss this papers in details, because of in this first time has mentioned about the 2D Boron based materials. Moreover, provided different structure with certain numbers of Boron atom and denoted point group symmetry, in addition to that derived structures has obtained stability, because of the presence of interaction piorbital out of a plane, a result of that formation of electron cloud sequent prevail stability. Besides, double layer Boron atomic layer highly stable, a consequence of that Boustani concluded that aforementioned contexts give an idea about possibilities for Boron polyhedral cluster having analogous properties to the Graphite [29]. After nine years in 2015, Andrew from Argonne laboratory ,USA has published an article commenting that, prepared first time grown atomically thin layer of Borophene sheets under ultra-high vacuum condition on top of $\mathrm{Ag}(111)$ substrate [30]. And claimed, at atomic cluster scale, pure form of Boron behavior like carbon, which created similar to simple planner and cage-like fullerenes [31] Bororphene shows metallic characteristics, in their papers have reported scanning tunneling microscopy images, which has shown that flakes kind of nature, follow Figure 2. In experimental and theoretical studies lead to systematic understanding structure of borophene, it has taken attention for $2 \mathrm{D}$ atom-thin sheet of boron found to be honeycomb structure like to Graphene, with hexagonal vacancies, which is highly stable that could possible to make boron nanotube with multiple sheets. The Piazza et al. have first time demonstrated experimental experience about hexagonal vacancies and it can vary with the deposition substrate [32]. In this present review article, it gives an idea about how borophene can overwhelm to graphene application, and moreover, we will address a few synthesis methods. Furthermore, in the case of application borophene has numerous applications, we have introduced a few of them, that includes energy conversion and storage, besides, that photocatalytic for $\mathrm{CO}_{2}$ reduction and water splitting. And the concerning hydrogen storage can be enhanced of storage capacity. Therefore, it gives a wide perspective towards new 2D material which is analogs properties to graphene or we can mention better than that.

\section{Synthesis of $\mathbf{2 D}$ atomic-thin layer Borophene}

The Borophene has synthesized first time by Andrew J. Mannix et al. in 2015 at the USA. The Borophene has grown on the top of $\operatorname{Ag}(111)$ as substrate under ultrahigh vacuum condition and used solid boron precursor. A substrate provided an inert surface to grown boron and maintained $450^{\circ \mathrm{C}}$ to $700^{\circ \mathrm{C}}$ substrate temperature. After deposited of boron, discovered that two phases have developed, that was a homogenous and striped phase, those depended on deposition rate, it has shown in figure 1 . The slow rate ended in the striped phase and higher rate dominated the homogenous phase [30]. Afterwards, they have done characterization and found gapless (metallic) characteristics, and this study motivated for scientist to work in future.

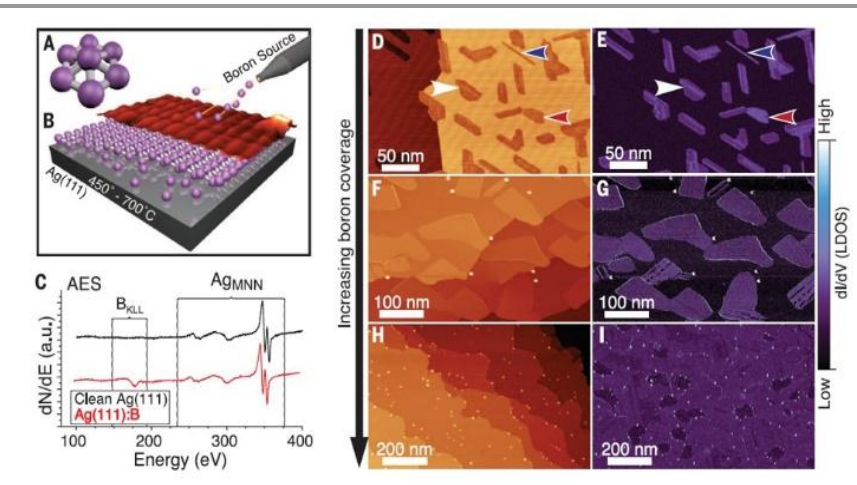

Scheme 1. Synthesis of 2D-thin layer borophene on top of $\mathrm{Ag}(111)$ substrate 
Figure 1A Showed that distorted $\mathrm{B}_{7}$ cluster, B) Growth set with atomic structure model and STM topography, C) This AES spectrum graph showed $\operatorname{Ag}(111)$ before and after deposition of boron. D) to I) illustrated STM topography and closed-loop $\mathrm{dI} / \mathrm{dV}$ images of borophene sheet left and right side respectively. In details, D and $\mathrm{E}$ showed that low coverage, $\mathrm{F}$ and $\mathrm{G}$ medium coverage, $\mathrm{H}$ and $\mathrm{I}$ has high coverage. Moreover, homogenous, striped, island and nanoribbon has shown with red, white and blue arrow correspondingly. Reproduced and reprint with permission [30].

Qing Zhong et al. came with similar substrate $\operatorname{Ag}(110)$, however, crystal lattice was different. And they have successfully prepared a single-layer atomic thin layer of borophene nanoribbon. Moreover, inferred about the substrate that, in beforehand $\operatorname{Ag}(111)$ crystal lattice substrate a growth of borophene formed triangular shape and edges are along three crystallographic orientation of $\operatorname{Ag}(111)$. In their investigation stated that self-assembly grows of borophene nanoribbon along [-110] direction of $\operatorname{Ag}(110)$, further appeared four periodic structure on borophene nanoribbon, additionally studied and confirmed properties with DFT( density functional theory) [33]. In 2013, Yuanyue Liu and co-workers explained the penetrating synthesis of 2D boron sheet with first-principle calculation. The 2D boron grown on the $\mathrm{Au}(111)$ and $\mathrm{Ag}(111)$ and $\mathrm{Cu}(111)$, this metals does not have reactivity, its leads formation compound with them. However, these metals provide extremely good for the nucleation process. Also, high nucleation $[34,35]$ rate barrier avoids the 3D formation of boron. Additionally, $\mathrm{Mg}$ offers reactivity with boron and forms metal borides, [36] in that case, a saturation of atoms impedes further growth of sheet [37]. Wenbin Li et al. have synthesized honeycomb borophene used as $\mathrm{Al}(111)$ substrate with help of molecular beam epitaxy(MBE) and concluded that, $\mathrm{Al}$ has stable substrate for boronphene formation. And noticed, effective charge transfer in $\mathrm{Al}$ with compared to $\mathrm{Ag}$, resultant claimed that it could help for intensification of electronic properties [38]. The substrate encouraged flexibility of the borophene has described by the Zhuhua Zhang et al. deposited on $\operatorname{Ag}(111)$ [39] and added elastic plate theory [40].

\section{Applications}

After, synthesized the borophene in 2015 , it has widely explored for energy application such energy storage and conversion. However, in current review article gives outlook and brief explanation about a few application of borophene.

\subsection{Energy Storage}

The modern world is going to suffer sooner because of the energy crisis, therefore, we need to have some lowcost and higher performance materials to enhance the energy capacity and outstanding cycle performance in energy storage devices. Recently, 2D based materials have been significantly growing. And 2D based materials electrode have shown remarkable electrochemical performance in energy storage capacity [41-43]. Graphene has played a pivotal role in energy storage, [44,45] as an excellent electrode system, however, beyond the graphene, also there are some 2D materials such as $\mathrm{MoS}_{2}$ [46,47], $\mathrm{WS}_{2}$ [48], $\mathrm{VS}_{2}$ [49] this are transition metal dichalcogenides, further in transition metal carbides so-called Mxene for an example $\mathrm{Mo}_{2} \mathrm{C}[50,51]$ and $\mathrm{Ti}_{3} \mathrm{C}_{2}$ [52] etc. Here, we have focused that borophene as a potential candidate for energy storage. Researchers already have done details study of properties for borophene and found one step ahead than graphene, therefore, this ideology has motivated to introduce this kind of materials for energy storage in different based ion batteries such $\mathrm{Li}, \mathrm{Na}$ and $\mathrm{Ca}$. Yang Zhang and co-worker Studied Li and $\mathrm{Na}$ ion adsorption and diffusion rate, used spin-polarized density function. And registered observation as $\mathrm{Li}$ atoms can bind effectively to borophene without energy barriers during the process, borophene and resultant adsorbed complexes exhibited the metallic characteristics, this necessary for an electrode. Borophene layer has corrugated structures, noticed that borophene itself might provide a channel facility for Li-adsorption. However, diffusion of Li-ion occurred in an uncorrugated direction, which has registered $10^{4}$ and $10^{5}$ faster than $\mathrm{MoS}_{2}$ and graphene respectively [53-55]. In 2016, has noticed the performance of Li-ion battery with two different structure, that was $\beta 12$ and $\chi 3$. Xiaoming Zhang et al. have improved $\mathrm{Li}$ and $\mathrm{Na}$ ion adsorption sites, also, found good electrical properties of before and after adsorption of host materials. And storage capacity has noticed significantly as $1984 \mathrm{mAhg}^{-1}$ in $\beta 12$ and 1240 $\mathrm{mAhg}^{-1}$ in $\chi^{3}$, therefore, it has much time better performance than current 2D materials electrode [56]. In Li-S batteries [57-61] has hurdle for the suppress the shuttle during a performance, after, investigated understand the lacking in the adsorption energy on the carbon surface of lithium polysulfides with compared to the electrolyte molecules, consequently, lithium polysulfide is easy to dissolve and transfer to the anode. The result of that effort has been taking to developing the anchoring group for enhancement of large adsorption energy in Li-S based batteries. To solve that problem, H.R. Jiang and co-worked came with borophene material and exhibited ultra-higher adsorbent capacity as anchoring material for $\mathrm{Li}-\mathrm{S}$ batteries. However, in borophene found a decomposition of Li-S during cycling, the effect of that 
loss of sulfur has noticed. The solution for that defective borophene, which has found effectively enhancing the adsorption energy, moreover, it stopped the decomposition of Li-S [62].

\begin{tabular}{|ccc|}
\hline Table 1. Use of borophene for the batteries & \\
\hline Borophene & $\begin{array}{c}\text { Theoretical } \\
\text { capacity }(\mathbf{m A h} / \mathbf{g})\end{array}$ & References \\
\hline p-doped & 1732 & {$[63]$} \\
Li0.75B & 1860 & {$[64]$} \\
$\begin{array}{c}\text { Novel boron } \\
\text { nanosheets }\end{array}$ & 3306 & {$[65]$} \\
$\begin{array}{c}\text { For Na-ion } \\
\text { batteries }\end{array}$ & 1240 & {$[66]$} \\
\hline
\end{tabular}

\subsection{Optoelectronic Applications}

The light modulation is a crucial aspect in the photonics [67] and optoelectronics field [68,69], therefore, we could regulate control of light in various channels such as optimizing materials attributes and effective novel materials. Nowadays, 2D materials have been dominating in the field of optoelectronic [70,7] area, because of unique optical and electronic [72,73] characteristics. So far there are many 2D materials, assuredly, its start with graphene, that has confirmed optical properties to a great extent. Yonder graphene [74], also registered 2D materials, for an examples $\mathrm{WS}_{2}$ [75], $\mathrm{MoSe}_{2}$ [76], $\mathrm{MoS}_{2}$ [77], this are transition metal dichalcogenides and it has notified extraordinary optical properties because we can optimize the band gaps from monolayer to bulk as high to low $\mathrm{E}_{\mathrm{g}}$ respectively. Furthermore, metal carbide [78] and metal nitride also found significantly great properties, notwithstanding, this is mostly more than two atoms, hereabouts, we are centring to a single element based 2D materials, currently, the new materials which have analogous features to graphene that is borophene as also attained extremely effective optoelectronic properties. Lyudmyla Adamska [79] et al. have confirmed fine-tuning the optoelectronic properties, two-dimensional boron synthesized of different crystal structure, its dependent on which substrate has used. While developing the borophene sheet typically, it uses to induced strain, accordingly, demonstrated two different structure that was $\beta_{12}$ and $\delta_{6}$, under appeared strain, resultant change in the band structure and charge density delocalization properties as tuneable because of few percentages of strain in borophene material. Furthermore, as we knew that changing band structure with monolayer to bulk, here also investigated by same research group that borophene as transparent electrode using density functional theory [80]. In 2017, J.W. Jiang and research group have observed that van der Waals heterostructure of tunable Schottky barrier and electronic properties in borophene/g-C2N and concluded that electronic properties of
borophene/gC2N Van der Waal heterostructure used first-principle calculations, noticed effective charge transport in heterostructure while applied external electric field. The consequence, of we, have an opportunity to tune carrier concentration and Schottky barrier with the help of tuning in the Van der Waal heterostructure [81]. This insight can be useful in nanophotonic and optoelectronic devices. L. Z. Liu et al. have concluded that effectively elimination of Schottky barrier as well as strong electrical field effect using monolayer borophene electrode [82]. This all facts have benefit for the electronic, nanophotonic and optoelectronic devices.

\subsection{Borophene Based Photocatalytic Activity}

Recently, this field has taken widely attention for water splitting to the production of hydrogen as fuel. In 1972, first demonstrated photocatalytic activity for water splitting by Honda-Fujishima using $\mathrm{TiO}_{2}$ as photocatalysis [83-86], however, $\mathrm{TiO}_{2}$ band gap comes under the UV region [87], therefore, resultant activity was not that great. To overcome this problem we should have semiconductor materials, which as bandgap in the visible region, afterwards many materials have discovered, nevertheless, now it has been great demanding for $2 \mathrm{D}$ based materials because of its tunable bandgap so that we could overcome the previous hurdle. Besides, 2D material has high surface area that provides active sites for catalytical activity [88]. The graphene $[89,90]$ is having high catalytical activity and showed for water splitting as well. However, currently, 2D transition metal dichalcogenides have been drastically highlighted for the photocatalytic activity due the long-time stability, tunable bandgap and available active sites for the reaction are existing effectively such $\mathrm{WO}_{3}[91], \mathrm{TiS}_{2}$ [92], $\mathrm{SnS}_{2}[93,94], \mathrm{WSe}_{2}[95,96]$, one more remarkable properties of this materials has conversion of indirect to direct band followed with bulk layer to monolayer correspondingly. Additionally, one more group of 2D materials based groups acquiring attention that is MXene [97]. So borophene also has similar properties likewise graphene, therefore, it has also as interesting photocatalytic activity. In 2016 Li Shi et al. [98] have demonstrated that boron monolayers as electrocatalyst properties for hydrogen evolution (HER) so-called water splitting, have used the first principle calculation to understand it. And concluded that boron monolayers show that approximately zero free energy $\left(\Delta \mathrm{G}_{\mathrm{H}}\right)$ for hydrogen adsorption because of it similar to the metallic properties such Pt. Moreover, proved the silver has a suitable efficient substrate for the HER activity because mismatch during the growth of borophene leads to enhancement of electrocatalytically activity. The borophene is weightless materials for the HER and Oxygen evolution reaction (OER), after doping of C, 
$\mathrm{N}, \mathrm{P}$, and $\mathrm{Li}$ element into borophene, the resultant effect has studied by Showkat H. Mir and co-worker, calculated the adsorption free energy for Oxygen and hydrogen. And resultants have examined that enhancing that catalytical activity, so its newly open window for lightweight 2D materials for HER and OER [99]. Recently in 2018 Chuangwei Liu et al. [95] claimed that borophene could be a metal-free catalyst. Aforementioned, many researchers [100-102] have proven that all this significant characteristics and evidence prove that highly possibility of having a remarkable change in HER and OER field because of the borophene as novel electrocatalyst.

\subsection{Hydrogen Storage}

In future hydrogen fuel will prominent source for energy, however, there are several challenges have been existing and researchers have been always fascinating to solve this obstacle. Suppose, if we are looking according to priorities safety is most important things in hydrogen storage, as we knew that energy density of hydrogen gas is excessive, hydrogen is very reactive with oxygen, it is an exothermic reaction. Moreover, solid-state hydrogen storage remains a major challenge. The metal hydrides [103,115] have extensively been used in hydrogen storage, however, unfortunately, stability and sluggish kinetics [104] are major drawbacks of this kind of materials. In the current situation, new families have been coming into hydrogen storage science, which has immense potential to overcome most of the problems that so far exists. The 2D materials are one of them have arrived in hydrogen storage and become useful in a great extent, there are numerous example such as graphene [105,106] has used majorly, however, nowadays, new family materials also been used such transition metal dichalcogenides [107,108] and alkali based 2D materials such as metal carbides and nitride [109]. The 2D materials are mechanically robust and it has a profoundly catalytical activity to enhance the kinetics for adsorption and desorption of hydrogen gas [110]. Moreover, it gives highly active sites that are high surface energy, that provides a large amount of adsorption of hydrogen gas. Recently, new material has been introduced in hydrogen storage, which as an analogue to graphene, that is borophene. In borophene found that has several steps ahead significant properties than graphene, and as we know that boron has extremely reactive with hydrogen, forms stable compounds. So far experimentally has not applied as expected, calculated storage capacity using first principle calculation, Chun-Sheng Liu et al. [111] have reported that reversible hydrogen storage in metallic hexagonal $\mathrm{B}_{36}$, explained the curvature and ionization. Borophene has coated with Li-metal to enhance the adsorptions and found adsorption energy has suitable for room temperature. Furthermore, several hydrogen molecules have measured by using negative differential resistance behavior at different bias voltage region. However, Li-atom has a problem that it adsorbs with low binding energy, therefore, Jianchuan wang et al. [112] have came with a solution, Calcium coated boron sheet, and registered that six molecules have captured at $0 \mathrm{~K}$ with binding energy as -0.20 to $-0.32 \mathrm{eV} / \mathrm{H}_{2}$. The mechanism of adsorption has appeared from not only the polarization effect but also the orbital hybridization and noticed $12.58 \mathrm{wt} \%$ gravimetric hydrogen density. Moreover, Ca-decorated [113-115] boron sheet at room after adsorption of hydrogen all structures were stable, this kind of materials could be useful for the vehicle application. Some of the researchers have tried with Na- decorated [116] as well instead of Li-atoms. Therefore borophene is having an immense potential for hydrogen storage, however, there are some challenges, to overcome that we need to have further modification.

\section{Conclusion}

Based on the concise illustrated above information about the borophene, we have inferred that it has enormous potential for the materials science field applications, notably for energy applications. Furthermore, borophene has not been examined much in an experimentally, because still, synthesis has to optimize. Nevertheless, recently for $\mathrm{Li}, \mathrm{Na}$ and $\mathrm{Mg}$ ionbased batteries has reported borophene is a potential candidate for the anode. The borophene has high surface energy, consequently, it has shown that high photocatalytic activity for water splitting. In the case of hydrogen storage, borophene has the most stable material than any other that so far available today. Sooner in future, will have tremendous attention in the materials science field, particularly in Li-ion batteries and hydrogen storage.

\section{Acknowledgment}

Author wants to thanks Mr. Vaishwik Patel for helping throughout the article. Dr Rajkumar wants to thanks for UGC, New Delhi for providing RJNF scholarship.

\section{Disclosure statement}

No potential conflict of interest was reported by the authors.

\section{References}

[1] Mas-Balleste, R., Gomez-Navarro, C., GomezHerrero, J., \& Zamora, F. (2011). 2D materials: to graphene and beyond. Nanoscale, 3(1), 20-30.

[2] Kannan, P. K., Late, D. J., Morgan, H., \& Rout, C. S. (2015). Recent developments in $2 \mathrm{D}$ layered 
inorganic nanomaterials for sensing. Nanoscale, 7(32), 13293-13312..

[3] Guo, Y., Xu, K., Wu, C., Zhao, J., \& Xie, Y. (2015). Surface chemical-modification for engineering the intrinsic physical properties of inorganic two-dimensional nanomaterials. Chemical Society Reviews, 44(3), 637-646.

[4] Gobbi, M., Orgiu, E., \& Samorì, P. (2018). When 2D materials meet molecules: opportunities and challenges of hybrid organic/inorganic van der Waals heterostructures.

Advanced Materials, 30(18), 1706103.

[5] Shein, I. R., \& Ivanovskii, A. L. (2013). Graphenelike nanocarbides and nanonitrides of $\mathrm{d}$ metals (MXenes): synthesis, properties and simulation. Micro \& Nano Letters, 8(2), 59-62.

[6] Lightcap, I. V., \& Kamat, P. V. (2012). Graphitic design: prospects of graphene-based nanocomposites for solar energy conversion, storage, and sensing. Accounts of chemical Research, 46(10), 2235-2243.

[7] Chandrasekaran, J., Nithyaprakash, D., Ajjan, K. B., Maruthamuthu, S., Manoharan, D., \& Kumar, S. (2011). Hybrid solar cell based on blending of organic and inorganic materials-An overview. Renewable and Sustainable Energy Reviews, 15(2), 1228-1238.

[8] Li, B., Gu, P., Feng, Y., Zhang, G., Huang, K., Xue, H., \& Pang, H. (2017). Ultrathin Nickel-Cobalt Phosphate 2D Nanosheets for Electrochemical Energy Storage under Aqueous/Solid-State Electrolyte. Advanced

Functional Materials, 27(12), 1605784.

[9] Mao, M., Hu, J., \& Liu, H. (2015). Graphene-based materials for flexible electrochemical energy storage. International Journal of Energy Research, 39(6), 727-740.

[10] Sreeprasad, T. S., \& Berry, V. (2013). How do the electrical properties of graphene change with its functionalization?. Small, 9(3), 341-350.

[11] Suk, J. W., Lee, W. H., Lee, J., Chou, H., Piner, R. D., Hao, Y., ... \& Ruoff, R. S. (2013). Enhancement of the electrical properties of graphene grown by chemical vapor deposition via controlling the effects of polymer residue. Nano letters, 13(4), 1462-1467.

[12] Frank, I. W., Tanenbaum, D. M., van der Zande, A. M., \& McEuen, P. L. (2007). Mechanical properties of suspended graphene sheets. Journal of Vacuum Science \& Technology B: Microelectronics and Nanometer Structures
Processing, Measurement, and Phenomena, 25(6), 2558-2561.

[13] Faccio, R., Denis, P. A., Pardo, H., Goyenola, C., \& Mombrú, A. W. (2009). Mechanical properties of graphene nanoribbons. Journal of Physics: Condensed Matter, 21(28), 285304.

[14] Balandin, A. A., Ghosh, S., Bao, W., Calizo, I., Teweldebrhan, D., Miao, F., \& Lau, C. N. (2008). Superior thermal conductivity of single-layer graphene. Nano letters, 8(3), 902-907.

[15] De Padova, P., Kubo, O., Olivieri, B., Quaresima, C., Nakayama, T., Aono, M., \& Le Lay, G. (2012). Multilayer silicene nanoribbons. Nano letters, 12(11), 5500-5503.

[16] Yamada-Takamura, Y., \& Friedlein, R. (2014). Progress in the materials science of silicene. Science and technology of advanced materials, 15(6), 064404.

[17] Ni, Z., Liu, Q., Tang, K., Zheng, J., Zhou, J., Qin, R., ... \& Lu, J. (2011). Tunable bandgap in silicene and germanene. Nano letters, 12(1), 113118.

[18] Mortazavi, B., Dianat, A., Cuniberti, G., \& Rabczuk, T. (2016). Application of silicene, germanene and stanene for $\mathrm{Na}$ or $\mathrm{Li}$ ion storage: $\mathrm{A}$ theoretical investigation. Electrochimica Acta, 213, 865-870.

[19] Dávila, M. E., Xian, L., Cahangirov, S., Rubio, A., \& Le Lay, G. (2014). Germanene: a novel twodimensional germanium allotrope akin to graphene and silicene. New Journal of Physics, 16(9), 095002.

[20] Carvalho, A., Wang, M., Zhu, X., Rodin, A. S., Su, H., \& Neto, A. H. C. (2016). Phosphorene: from theory to applications. Nature Reviews Materials, 1(11), 16061.

[21] Woomer, A. H., Farnsworth, T. W., Hu, J., Wells, R. A., Donley, C. L., \& Warren, S. C. (2015). Phosphorene: synthesis, scale-up, and quantitative optical spectroscopy. ACS nano, 9(9), 8869-8884.

[22] Wang, Y. P., Ji, W. X., Zhang, C. W., Li, P., Li, F., Ren, M. J., ... \& Wang, P. J. (2016). Controllable band structure and topological phase transition in two-dimensional hydrogenated arsenene. Scientific reports, 6, 20342.

[23] Kecik, D., Durgun, E., \& Ciraci, S. (2016). Optical properties of single-layer and bilayer arsenene phases. Physical Review B, 94(20), 205410.

[24] Sharma, S., Kumar, S., \& Schwingenschlögl, U. (2017). Arsenene and antimonene: two- 
dimensional materials with high thermoelectric figures of merit. Physical Review Applied, 8(4), 044013.

[25] Wilson, J. A., \& Yoffe, A. D. (1969). The transition metal dichalcogenides discussion and interpretation of the observed optical, electrical and structural properties. Advances in Physics, 18(73), 193-335.

[26] Wilson, J. A., Di Salvo, F. J., \& Mahajan, S. (1975). Charge-density waves and superlattices in the metallic layered transition metal dichalcogenides. Advances in Physics, 24(2), 117201.

[27] Manzeli, S., Ovchinnikov, D., Pasquier, D., Yazyev, O. V., \& Kis, A. (2017). 2D transition metal dichalcogenides. Nature Reviews Materials, 2(8), 17033.

[28] Boustani, I. (1997). New quasi-planar surfaces of bare boron. Surface science, 370(2-3), 355-363.

[29] Saito, R., Fujita, M., Dresselhaus, G., \& Dresselhaus, U. M. (1992). Electronic structure of chiral graphene tubules. Applied physics letters, 60(18), 2204-2206.

[30] Mannix, A. J., Zhou, X. F., Kiraly, B., Wood, J. D., Alducin, D., Myers, B. D., \& Yacaman, M. J. (2015). Synthesis of borophenes: Anisotropic, two-dimensional boron polymorphs. Science, 350(6267), 1513-1516.

[31] Seifert, G., Heine, T., \& Fowler, P. W. (2001). Inorganic nanotubes and fullerenes. The European Physical Journal D-Atomic, Molecular, Optical and Plasma Physics, 16(1), 341-343.

[32] Piazza, Z. A., Hu, H. S., Li, W. L., Zhao, Y. F., Li, J., \& Wang, L. S. (2014). Planar hexagonal B 36 as a potential basis for extended single-atom layer boron sheets. Nature communications, 5, 3113.

[33] Zhong, Q., Kong, L., Gou, J., Li, W., Sheng, S., Yang, S., ... \& Chen, L. (2017). Synthesis of borophene nanoribbons on $\mathrm{Ag}$ (110) surface. Physical Review Materials, 1(2), 021001.

[34] Jun, Y. S., Kim, D., \& Neil, C. W. (2016). Heterogeneous nucleation and growth of nanoparticles at environmental interfaces. Accounts of chemical research, 49(9), 1681-1690.

[35] Resta, V., Afonso, C. N., Piscopiello, E., \& Van Tendeloo, G. (2009). Role of substrate on nucleation and morphology of gold nanoparticles produced by pulsed laser deposition. Physical Review B, 79(23), 235409.
[36] Mavel, G., Escard, J., Costa, P., \& Castaing, J. (1973). ESCA surface study of metal borides. Surface Science, 35, 109-116.

[37] Liu, Y., Penev, E. S., \& Yakobson, B. I. (2013). Probing the synthesis of two-dimensional boron by first-principles computations. Angewandte Chemie International Edition, 52(11), 3156-3159.

[38] Li, W., Kong, L., Chen, C., Gou, J., Sheng, S., Zhang, W., ... \& Wu, K. (2018). Experimental realization of honeycomb borophene. Science bulletin, 63(5), 282-286.

[39] Zhang, Z., Mannix, A. J., Hu, Z., Kiraly, B., Guisinger, N. P., Hersam, M. C., \& Yakobson, B. I. (2016). Substrate-induced nanoscale undulations of borophene on silver. Nano letters, 16(10), 66226627.

[40] Sutti, M. (2015). Elastic Theory of Plates.

[41] Zhang, X., Huisman, E. H., Gurram, M., Browne, W. R., van Wees, B. J., \& Feringa, B. L. (2014). Supramolecular Chemistry on Graphene Field-Effect Transistors. Small, 10(9), 1735-1740.

[42] Wang, H., Feng, H., \& Li, J. (2014). Graphene and graphene-like layered transition metal dichalcogenides in energy conversion and storage. Small, 10(11), 2165-2181.

[43] Li, H., Shi, Y., Chiu, M. H., \& Li, L. J. (2015). Emerging energy applications of two-dimensional layered transition metal dichalcogenides. Nano Energy, 18, 293-305.

[44] Pumera, M. (2011). Graphene-based nanomaterials for energy storage. Energy \& Environmental Science, 4(3), 668-674.

[45] Brownson, D. A., Kampouris, D. K., \& Banks, C. E. (2011). An overview of graphene in energy production and storage applications. Journal of Power Sources, 196(11), 4873-4885.

[46] Wang, T., Chen, S., Pang, H., Xue, H., \& Yu, Y. (2017). $\mathrm{MoS}_{2}$-based nanocomposites for electrochemical energy storage. Advanced Science, 4(2), 1600289.

[47] Wang, J., Liu, J., Chao, D., Yan, J., Lin, J., \& Shen, Z. X. (2014). Self-Assembly of Honeycomblike $\mathrm{MoS}_{2}$ Nanoarchitectures Anchored into Graphene Foam for Enhanced Lithium-Ion Storage. Advanced Materials, 26(42), 7162-7169.

[48] Lei, T., Chen, W., Huang, J., Yan, C., Sun, H., Wang, C., ... \& Xiong, J. (2017). Multi-Functional Layered $\mathrm{WS}_{2}$ Nanosheets for Enhancing the Performance of Lithium-Sulfur Batteries. Advanced Energy Materials, 7(4), 1601843.

[49] He, P., Yan, M., Zhang, G., Sun, R., Chen, L., An, Q., \& Mai, L. (2017). Layered $\mathrm{VS}_{2}$ Nanosheet- 
Based Aqueous Zn Ion Battery Cathode. Advanced Energy Materials, 7(11), 1601920.

[50] Shi, M., Zhao, L., Song, X., Liu, J., Zhang, P., \& Gao, L. (2016). Highly conductive $\mathrm{Mo}_{2} \mathrm{C}$ nanofibers encapsulated in ultrathin $\mathrm{MnO}_{2}$ nanosheets as a self-supported electrode for highperformance capacitive energy storage. ACS applied materials \& interfaces, 8(47), 3246032467.

[51] Zhu, J., Sakaushi, K., Clavel, G., Shalom, M., Antonietti, M., \& Fellinger, T. P. (2015). A general salt-templating method to fabricate vertically aligned graphitic carbon nanosheets and their metal carbide hybrids for superior lithium ion batteries and water splitting. Journal of the American Chemical Society, 137(16), 5480-5485.

[52] Li, B., Zhang, D., Liu, Y., Yu, Y., Li, S., \& Yang, S. (2017). Flexible $\mathrm{Ti}_{3} \mathrm{C}_{2}$ MXene-lithium film with lamellar structure for ultrastable metallic lithium anodes. Nano energy, 39, 654-661.

[53] Zhang, Y., Wu, Z. F., Gao, P. F., Zhang, S. L., $\&$ Wen, Y. H. (2016). Could borophene be used as a promising anode material for high-performance lithium ion battery? ACS applied materials \& interfaces, 8(34), 22175-22181.

[54] Uthaisar, C., \& Barone, V. (2010). Edge effects on the characteristics of $\mathrm{Li}$ diffusion in graphene. Nano letters, 10(8), 2838-2842.

[55] Li, Y., Wu, D., Zhou, Z., Cabrera, C. R., \& Chen, Z. (2012). Enhanced $\mathrm{Li}$ adsorption and diffusion on MoS2 zigzag nanoribbons by edge effects: a computational study. The journal of physical chemistry letters, 3(16), 2221-2227.

[56] Zhang, X., Hu, J., Cheng, Y., Yang, H. Y., Yao, Y., \& Yang, S. A. (2016). Borophene as an extremely high capacity electrode material for $\mathrm{Li}$ ion and Na-ion batteries. Nanoscale, 8(33), 1534015347.

[57] Bruce, P. G., Freunberger, S. A., Hardwick, L. J., \& Tarascon, J. M. (2012). Li-O 2 and $\mathrm{Li}-\mathrm{S}$ batteries with high energy storage. Nature materials, 11(1), 19.

[58] Ji, X., \& Nazar, L. F. (2010). Advances in LiS batteries. Journal of Materials Chemistry, 20(44), 9821-9826.

[59] Li, Y. J., Fan, J. M., Zheng, M. S., \& Dong, Q. F. (2016). A novel synergistic composite with multi-functional effects for high-performance $\mathrm{Li}-\mathrm{S}$ batteries. Energy \& Environmental Science, 9(6), 1998-2004.

[60] Zheng, S., Yi, F., Li, Z., Zhu, Y., Xu, Y., Luo, C., \& Wang, C. (2014). Copper-stabilized sulfur- microporous carbon cathodes for $\mathrm{Li}-\mathrm{S}$ batteries. Advanced Functional Materials, 24(26), 41564163.

[61] Powar, N. S., Patel, V. J., Pagare, P. K., \& Pandav, R. S. (2019). Cu Nanoparticle: Synthesis, Characterization and Application. Chemical Methodologies, 3(4), 457-480.

[62] Jiang, H. R., Shyy, W., Liu, M., Ren, Y. X., \& Zhao, T. S. (2018). Borophene and defective borophene as potential anchoring materials for lithium-sulfur batteries: a first-principles study. Journal of Materials Chemistry A, 6(5), 21072114.

[63] Chen, H., Zhang, W., Tang, X. Q., Ding, Y. H., Yin, J. R., Jiang, Y., \& Jin, H. (2018). First principles study of P-doped borophene as anode materials for lithium ion batteries. Applied Surface Science, 427, 198-205.

[64] Jiang, H. R., Lu, Z., Wu, M. C., Ciucci, F., \& Zhao, T. S. (2016). Borophene: a promising anode material offering high specific capacity and high rate capability for lithium-ion batteries. Nano Energy, 23, 97-104.

[65] Rao, D., Zhang, L., Meng, Z., Zhang, X., Wang, Y., Qiao, G., \& Lu, R. (2017). Ultrahigh energy storage and ultrafast ion diffusion in borophene-based anodes for rechargeable metal ion batteries. Journal of Materials Chemistry A, 5(5), 2328-2338.

[66] Liu, J., Zhang, C., Xu, L., \& Ju, S. (2018). Borophene as a promising anode material for sodium-ion batteries with high capacity and high rate capability using DFT. RSC advances, 8(32), 17773-17785.

[67] Kildishev, A. V., Boltasseva, A., \& Shalaev, V. M. (2013). Planar photonics with metasurfaces. Science, 339(6125), 1232009.

[68] Lu, H. H., Chen, G. L., Chuang, Y. W., Tsai, C. C., \& Chuang, C. P. (2006). Improvement of radio-on-multimode fiber systems based on light injection and optoelectronic feedback techniques. Optics Communications, 266(2), 495-499.

[69] Opeyemi, O., Louis, H., Oparab, C., Funmilayo, O., Magu, T. (2019). Porphyrin and Phthalocyanines-Based Solar Cells: Fundamental Mechanisms and Recent Advances. Advanced Journal of Chemistry, Section A: Theoretical, Engineering and Applied Chemistry, 2(Issue 1, pp. 1-93.), 21-44.

[70] Wang, Q. H., Kalantar-Zadeh, K., Kis, A., Coleman, J. N., \& Strano, M. S. (2012). Electronics and optoelectronics of two-dimensional transition 
metal dichalcogenides. Nature nanotechnology, 7(11), 699.

[71] Huo, N., Yang, Y., \& Li, J. (2017). Optoelectronics based on 2D TMDs and heterostructures. Journal of Semiconductors, 38(3), 031002.

[72] Yazyev, O. V., \& Louie, S. G. (2010). Electronic transport in polycrystalline graphene. Nature materials, 9(10), 806.

[73] Eda, G., Fanchini, G., \& Chhowalla, M. (2008). Large-area ultrathin films of reduced graphene oxide as a transparent and flexible electronic material. Nature nanotechnology, 3(5), 270.

[74] Ponraj, J. S., Xu, Z. Q., Dhanabalan, S. C., Mu, H., Wang, Y., Yuan, J. \& Zhang, Y. (2016). Photonics and optoelectronics of two-dimensional materials beyond graphene. Nanotechnology, 27(46), 462001.

[75] Kim, H. C., Kim, H., Lee, J. U., Lee, H. B., Choi, D. H., Lee, J. H., \& Lee, S. W. (2015). Engineering optical and electronic properties of $\mathrm{WS}_{2}$ by varying the number of layers. ACS nano, 9(7), 6854-6860.

[76] Ko, P. J., Abderrahmane, A., Kim, N. H., \& Sandhu, A. (2017). High-performance nearinfrared photodetector based on nano-layered MoSe2. Semiconductor Science and Technology, 32(6), 065015.

[77] Splendiani, A., Sun, L., Zhang, Y., Li, T., Kim, J., Chim, C. Y., \& Wang, F. (2010). Emerging photoluminescence in monolayer $\mathrm{MoS}_{2}$. Nano letters, 10(4), 1271-1275.

[78] Anasori, B., Lukatskaya, M. R., \& Gogotsi, Y. (2017). 2D metal carbides and nitrides (MXenes) for energy storage. Nature Reviews Materials, 2(2), 16098.

[79] Adamska, L., \& Sharifzadeh, S. (2017). Finetuning the optoelectronic properties of freestanding borophene by strain. ACS Omega, 2(11), 82908299.

[80] Adamska, L., Sadasivam, S., Foley IV, J. J., Darancet, P., \& Sharifzadeh, S. (2018). Firstprinciples investigation of borophene as a monolayer transparent conductor. The Journal of Physical Chemistry C, 122(7), 4037-4045.

[81] Jiang, J. W., Wang, X. C., Song, Y., \& Mi, W. B. (2018). Tunable Schottky barrier and electronic properties in borophene/g- $\mathrm{C}_{2} \mathrm{~N}$ van der Waals heterostructures. Applied Surface Science, 440, 42-46.
[82] Liu, L. Z., Xiong, S. J., \& Wu, X. L. (2016). Monolayer borophene electrode for effective elimination of both the Schottky barrier and strong electric field effect. Applied Physics Letters, 109(6), 061601.

[83] Fujishima, A., \& Honda, K. (1972). Electrochemical photolysis of water at a semiconductor electrode. nature, 238(5358), 37.

[84] Salavati, H., Teimouri, A., Kazemi, S. (2017). Synthesis and Characterization of Novel Composite-Based Phthalocyanine Used as Efficient Photocatalyst for the Degradation of Methyl Orange. Chemical Methodologies, 1(Issue 1. pp. 1-86), 12-27.

[85] Salavati, H., Teimouri, A., Kazemi, S. (2017). Investigation of Photocatalytic Performance of Keggin Type Heteropolyacid in Degradation of Methylene Blue. Chemical Methodologies, 1(Issue 2. pp. 87-193), 145-158.

[86] Madadi, Z., Soltanieh, M., Bagheri Lotfabad, T., Nazari, S. (2019). Green synthesis of titanium dioxide nanoparticles with Glycyrrhiza glabra and their photocatalytic activity. Asian Journal of Green Chemistry,

[87] Jefri, S., Abdullah, A., Muhamad, E. (2019). Response surface methodology: photodegradation of methyl orange by $\mathrm{CuO} / \mathrm{ZnO}$ under $\mathrm{UV}$ light irradiation. Asian Journal of Green Chemistry, 3(Issue 2. pp. 125-287), 271-287.

[88] Zhang, X., Zhang, Z., Wu, D., Zhang, X., Zhao, X., \& Zhou, Z. (2018). Computational screening of $2 \mathrm{D}$ materials and rational design of heterojunctions for water splitting photocatalysts. Small Methods, 2(5), 1700359.

[89] Niu, P., Zhang, L., Liu, G., \& Cheng, H. M. (2012). Graphene-like carbon nitride nanosheets for improved photocatalytic activities. Advanced Functional Materials, 22(22), 4763-4770.

[90] Xiang, Q., Yu, J., \& Jaroniec, M. (2011). Enhanced photocatalytic $\mathrm{H}_{2}$-production activity of graphene-modified titania nanosheets. Nanoscale, 3(9), 3670-3678.

[91] Do, Y. R., Lee, W., Dwight, K., \& Wold, A. (1994). The effect of $\mathrm{WO}_{3}$ on the photocatalytic activity of $\mathrm{TiO}_{2}$. Journal of Solid State Chemistry, 108(1), 198-201.

[92] Gupta, U., Rao, B. G., Maitra, U., Prasad, B. E., \& Rao, C. N. R. (2014). Visible-Light-Induced Generation of $\mathrm{H}_{2}$ by Nanocomposites of FewLayer $\mathrm{TiS}_{2}$ and $\mathrm{TaS}_{2}$ with CdS Nanoparticles. Chemistry-An Asian Journal, 9(5), 1311-1315. 
[93] Zhuang, H. L., \& Hennig, R. G. (2013). Theoretical perspective of photocatalytic properties of single-layer $\mathrm{SnS}_{2}$. Physical Review B, 88(11), 115314.

[94] Yu, J., Xu, C. Y., Ma, F. X., Hu, S. P., Zhang, Y. W., \& Zhen, L. (2014). Monodisperse $\mathrm{SnS}_{2}$ nanosheets for high-performance photocatalytic hydrogen generation. ACS applied materials \& interfaces, 6(24), 22370-22377.

[95] Singh, A. K., Mathew, K., Zhuang, H. L., \& Hennig, R. G. (2015). Computational screening of 2D materials for photocatalysis. The journal of physical chemistry letters, 6(6), 1087-1098.

[96] Wang, Y., Zhao, S., Wang, Y., Laleyan, D. A., Wu, Y., Ouyang, B., \& Mi, Z. (2018). Wafer-scale synthesis of monolayer $\mathrm{WSe}_{2}$ : a multi-functional photocatalyst for efficient overall pure water splitting. Nano Energy, 51, 54-60.

[97] Ran, J., Gao, G., Li, F. T., Ma, T. Y., Du, A., \& Qiao, S. Z. (2017). $\mathrm{Ti}_{3} \mathrm{C}_{2}$ MXene co-catalyst on metal sulfide photo-absorbers for enhanced visiblelight photocatalytic hydrogen production. Nature communications, 8, 13907.

[98] Shi, L., Ling, C., Ouyang, Y., \& Wang, J. (2017). High intrinsic catalytic activity of twodimensional boron monolayers for the hydrogen evolution reaction. Nanoscale, 9(2), 533-537.

[99] Mir, S. H., Chakraborty, S., Jha, P. C., Wärnå, J., Soni, H., Jha, P. K., \& Ahuja, R. (2016). Twodimensional boron: Lightest catalyst for hydrogen and oxygen evolution reaction. Applied Physics Letters, 109(5), 053903.

[100] Liu, C., Dai, Z., Zhang, J., Jin, Y., Li, D., \& Sun, C. (2018). Two-dimensional boron sheets as metal-free catalysts for hydrogen evolution reaction. The Journal of Physical Chemistry C, 122(33), 19051-19055.

[101] Chen, Y., Yu, G., Chen, W., Liu, Y., Li, G. D., Zhu, P., \& Li, H. (2017). Highly active, nonprecious electrocatalyst comprising borophene subunits for the hydrogen evolution reaction. Journal of the American Chemical Society, 139(36), 12370-12373.

[102] Park, H., Encinas, A., Scheifers, J. P., Zhang, Y., \& Fokwa, B. P. (2017). Boron-Dependency of Molybdenum Boride Electrocatalysts for the Hydrogen Evolution Reaction. Angewandte Chemie International Edition, 56(20), 5575-5578.

[103] Sakintuna, B., Lamari-Darkrim, F., \& Hirscher, M. (2007). Metal hydride materials for solid hydrogen storage: a review. International journal of hydrogen energy, 32(9), 1121-1140.
[104] Orimo, S. I., Nakamori, Y., Eliseo, J. R., Züttel, A., \& Jensen, C. M. (2007). Complex hydrides for hydrogen storage. Chemical Reviews, 107(10), 4111-4132.

[105] Chen, P., Xiong, Z., Luo, J., Lin, J., \& Tan, K. L. (2002). Interaction of hydrogen with metal nitrides and imides. Nature, 420(6913), 302.

[106] Vajo, J. J., \& Olson, G. L. (2007). Hydrogen storage in destabilized chemical systems. Scripta Materialia, 56(10), 829-834.

[107] Tozzini, V., \& Pellegrini, V. (2013). Prospects for hydrogen storage in graphene. Physical Chemistry Chemical Physics, 15(1), 80-89.

[108] Patchkovskii, S., John, S. T., Yurchenko, S. N., Zhechkov, L., Heine, T., \& Seifert, G. (2005). Graphene nanostructures as tunable storage media for molecular hydrogen. Proceedings of the National Academy of Sciences, 102(30), 1043910444.

[109] Pumera, M., Sofer, Z., \& Ambrosi, A. (2014). Layered transition metal dichalcogenides for electrochemical energy generation and storage. Journal of Materials Chemistry A, 2(24), 89818987.

[110] Gao, Y. P., Wu, X., Huang, K. J., Xing, L. L., Zhang, Y. Y., \& Liu, L. (2017). Two-dimensional transition metal diseleniums for energy storage application: a review of recent developments. CrystEngComm, 19(3), 404-418.

[111] Hu, Q., Wang, H., Wu, Q., Ye, X., Zhou, A., Sun, D., \& He, J. (2014). Two-dimensional $\mathrm{Sc}_{2} \mathrm{C}$ : A reversible and high-capacity hydrogen storage material predicted by first-principles calculations. International Journal of Hydrogen Energy, 39(20), 10606-10612.

[112] Martin, M., Gommel, C., Borkhart, C., \& Fromm, E. (1996). Absorption and desorption kinetics of hydrogen storage alloys. Journal of Alloys and Compounds, 238(1-2), 193-201.

[113] Liu, C. S., Wang, X., Ye, X. J., Yan, X., \& Zeng, Z. (2014). Curvature and ionization-induced reversible hydrogen storage in metalized hexagonal B36. The Journal of chemical physics, 141(19), 194306.

[114] Wang, J., Du, Y., \& Sun, L. (2016). Cadecorated novel boron sheet: a potential hydrogen storage medium. International Journal of Hydrogen Energy, 41(10), 5276-5283.

[115] Chen, X., Wang, L., Zhang, W., Zhang, J., \& Yuan, Y. (2017). Ca-decorated borophene as potential candidates for hydrogen storage: A first- 
principle study. International Journal of Hydrogen Energy, 42(31), 20036-20045.

[116] Wang, L., Chen, X., Du, H., Yuan, Y., Qu, H., $\&$ Zou, M. (2018). First-principles investigation on hydrogen storage performance of $\mathrm{Li}, \mathrm{Na}$ and $\mathrm{K}$ decorated borophene. Applied Surface Science, 427, 1030-1037.

How to cite this manuscript: Niket Powar, Rajkumar Pandav, Overview of Borophene as a

Potential Candidate in 2D Materials Science for the Energy Applications, Journal of Chemical Reviews (J. Chem. Rev. ), 2019, 1(4), 271-281. 